\title{
DESCOLONIZAR LAS LUCHAS: LA PROPUESTA DEL FEMINISMOCOMUNITARIO
}

\author{
Julieta Paredes Carvajal* \\ FeminismoComunitario de Abya Yala
}

\begin{abstract}
RESUMEN
El FeminismoComunitario tiene la característica de llegar y conmover a los pueblos, especialmente a las mujeres de los pueblos de Abya Yala' (América). Porque nació de los pueblos de Bolivia, que luchamos por nuestra liberación y por construir el Vivir bien; de la humanidad, de la naturaleza y del planeta. Este movimiento también nace desde la necesidad de las mujeres de los pueblos originarios, y de la clase trabajadora, que queremos nombrar nuestras prácticas políticas y nombrar nuestros sueños de vida. Nombrar, conceptualizar, crear argumentos, elaborar nuestros discursos y desmitificar el manejo de la palabra escrita es una tarea profundamente revolucionaria, esto de creer en nosotras y nosotros. Tomar la palabra escrita, para que escribamos y quede documento de lo que pensamos y soñamos, es ser parte de la memoria explícita del Planeta. Vivimos en un mundo con dominio de la cultura y el pensamiento occidental, la cultura eurooccidental sigue siendo el centro de la hegemonía, del poder en el mundo. Necesitamos como humanidad descolonizar nuestras vidas, nuestros cuerpos, nuestras espiritualidades y sobre todo nuestras luchas de este dominio. De aquí nace el FeminismoComunitario, que descoloniza nuestras luchas como mujeres en el territorio de Bolivia y nos posicionamos, de cara al mundo, junto a todas nuestras hermanas de los distintos territorios en Abya Yala, que son tejidos de luchadoras, en el continente como una gran manta, un gran awayo,
\end{abstract}

* Integrante fundadora de la agrupación feminista boliviana Mujeres Creando 1990, integrante fundadora de la comunidad Mujeres Creando comunidad 2002, Integrante de la Asamblea de FeminismoComunitario.

1 Abya Yala es el nombre dado al continente americano por el pueblo Kuna de Panamá y Colombia antes de la llegada de Cristóbal Colón y los europeos. Literalmente significaría "tierra en plena madurez" o "tierra de sangre vital". Los pueblos originarios usan este nombre como una reivindicación, política, discursiva y descolonizadora. 
que abriga las esperanzas y sueños de la Madre Naturaleza y de la humanidad que somos sus hijas e hijos.

Palabras clave: FeminismoComunitario. Bolivia. Feminismo. Patriarcado. Entronque patriarcal.

\title{
DESCOLONIZAR AS LUTAS: A PROPOSTA DO FEMINISMOCOMU- NITARIO
}

\section{RESUMO}

O FeminismoComunitário tem a característica de alcançar e mover os povos, especialmente as mulheres das cidades de Abya Yala (América). Porque ele nasceu do povo da Bolívia, que luta por sua libertação e pela construção do Bem-Viver; da humanidade, da natureza e do planeta. Esse movimento, também nascido da necessidade, das mulheres dos povos originários e da classe trabalhadora que quer dar nome às suas práticas políticas e sonhos de vida. Nomear, conceituar, criando argumentos, fazendo os nossos discursos e desmistificando a gestão da palavra escrita é uma tarefa profundamente revolucionária, a de acreditar em nós e por nós. Tomar a palavra escrita, para que escrevamos e permaneçamos como um documento do que pensamos e sonhamos é fazer parte da memória explícita do Planeta. Vivemos em um mundo dominado pela cultura e pelo pensamento ocidentais, a cultura euro-ocidental continua sendo o centro da hegemonia, do poder no mundo. Precisamos, como humanidade, descolonizar nossas vidas, nossos corpos, nossas espiritualidades e, acima de tudo, nossas lutas neste domínio. Daí o FeminismoComunitário descolonizar as nossas lutas como mulheres no território da Bolívia e nos posicionando, de frente para o mundo, com todas as nossas irmãs nos diferentes territórios em Abya Yala, que são tecidos de lutadores, no continente nasce um grande cobertor, um grande awayo, que abriga as esperanças e sonhos da Mãe Natureza e da humanidade que são suas filhas e filhos. Palavras-chave: FeminismoComunitário. Bolívia. Feminismo. Patriarcado. Relação patriarcal.

\section{DECOLONIZE THE STRUGGLES: THE COMMUNITARIAN FEMINISM PROPOSAL}

\author{
ABSTRACT \\ Communitarian Feminism has the characteristic of reaching and \\ moving the peoples, especially the women of the towns of Abya
}


Yala (America). Because it was born of the people of Bolivia, that we fight for our liberation and for building the Living well; of humanity, nature and the planet. This movement, also born from the need of the women of the original peoples, and of the working class that we want to name our political practices and name our dreams of life. Naming, conceptualizing, creating arguments, elaborating our discourses and demystifying the handling of the written word is a profoundly revolutionary task, that of believing in us and us. To take the written word, so that we write and remain a document of what we think and dream, is to be part of the explicit memory of the Planet. We live in a world dominated by Western culture and thought, the Euro-Western culture remains the center of hegemony, of power in the world. We need as a humanity to decolonize our lives, our bodies, our spiritualities and above all our struggles in this domain. This is the origin of Communitarian Feminism, which decolonizes our struggles as women in the territory of Bolivia and positions us, facing the world, together with all our sisters from the different territories in Abya Yala, which are woven as fighters, in the continent as a great blanket, a great awayo, that harbors the hopes and dreams of Mother Nature and humanity that are her daughters and sons.

Keywords: Communitarian Feminism. Bolivia. Feminism. Patriarchy. Patriarchal junction.

\section{LA ENERGÍA CONVOCANTE DEL FEMINISMOCOMUNITARIO DE ABYA YALA}

Es el FeminismoComunitario quien ha propuesto un espacio de discusión y de desafíos descolonizadores, que convocan a repensarnos, como cuerpos marginalizados, en todos los espacios de la existencia. Pero a la vez cuerpos rebeldes. Convocamos a un espacio de reflexión, no de competitividad.

En este camino nos parece necesario posicionarnos ante las discusiones actuales del feminismo y decimos que es difícil revolucionar la construcción de saberes, solo a partir de la discusión de la categoría de género, que es el espacio de discusión de un feminismo neoliberal y liberal. Es un espacio sumamente reducido, mezquino, respecto de toda la potencialidad de las mujeres. Es mezquino porque el sistema patriarcal es reduccionista, ignorante y depredador de todo lo nuevo que se crea. El cuestionamiento epistémico desde nuestros pueblos 
tiene que plantarse de manera que sea un dialogo útil para las luchas de los pueblos del mundo.

Las reflexiones de algunas hermanas indígenas - creo negras también - se ubican dentro de lo que se denomina como reflexiones sobre la colonialidad del género, que lo entiendo, como la mirada racista del género o también leer el género a partir de la colonización de 1492. Ciertamente es un comienzo y así es como lo han venido haciendo algunas hermanas indígenas en el continente, sin embargo, las estrategias fagocitadoras de las academias funcionan de inmediato y las reflexiones de nuestras hermanas indígenas son absorbidas por un ecofeminismo, por un feminismo de la diferencia, o son colocadas en feminismos decoIoniales o postcoloniales. Nosotras creemos que no dejan de ser estos, compartimentos estancos, controlados por el pensamiento hegemónico y colonial de occidente.

Aun la estrategia epistémica del feminismo comunitario, que luego vamos a exponer, no se salva de la intencionalidad fagocitaria de la academia, de la manipulaión de las ONGs, y de la depredación de plagiadoras que nunca faltan. Sin embargo, a pesar de estos intentos, no pueden con nosotras, pues no es fácil digerir un feminismo comunitario que, cuando se ubica en el feminismo, es para cuestionar al propio feminismo. Les cuestionamos en su supuesta titularidad y vanguardia de las luchas de las mujeres en el mundo. O lo que supuestamente sería lo políticamente correcto para las mujeres en el mundo. Por eso hoy el FeminismoComunitario es una energía que convoca a la reflexión, a la creación y a la sanación del mundo. Lejos de prácticas lapidarias y competitivas, entre mujeres, convocando a construir comunidad

\section{EL FEMINISMO, COMO LUCHA DE LAS MUJERES, NO ES EXCLUSI- VIDAD DE LAS EUROPEO-OCCIDENTALES}

Europa cree que es poseedora de todos los conocimientos, los pensamientos y luchas validas, de la humanidad, por ejemplo; si hablamos de participación y organización política del pueblo entonces, inmediatamente, le llaman; Democracia. Si hablamos de historia de nuestros pueblos del mundo, inmediatamente se habla en Europa de "historia universal" y solo se refieren a la historia de Europa. Si hablamos de palabras como 
progreso, civilización, tecnología, política, cultura, arte, razón, entre otros, el referente, es Europa occidental. Por lo tanto, si hablamos, de lucha de las mujeres, el feminismo europeo es el referente mundial.

Es decir que, por desgracia para el resto de las mujeres luchadoras del mundo, que somos pertenecientes a otras culturas, formas de pensamiento y conocimientos, tenemos que aguantar sobre nuestra historia de luchas la comparación descalificadora, con occidente. Mujeres constructoras de las historias de sus pueblos, por desgracia, son ignoradas porque hay un espacio de significados planteados hegemónicamente. Feminismo en el mundo hoy significa, para bien y para mal, lucha de las mujeres que empieza en Europa.

Entonces es necesario entender el origen de la palabra y la relación con nuestras luchas. A partir de 1492, por la invasión colonial a nuestros territorios, se han dado relaciones coloniales entre Europa y Abya Yala, esto significa, entre otras cosas que, en Europa piensan que inventaron las luchas de las mujeres contra el patriarcado, creen que pueden enseñar al mundo modelos de sociedad y modelos de cómo luchar para conseguir la sociedad que se desea.

Es verdad que la palabra y sonido de, feminismo - y la forma como se usa la palabra - vino de Europa, como una acumulación política, desde la revolución francesa en 1789, pero ellas, las europeas, no inventaron las luchas de las mujeres en el mundo, ni contra el patriarcado ni contra las opresiones. Nosotras tenemos nuestra propia versión de luchar. Tampoco podemos decir que Europa es el modelo de sociedad al que el mundo pueda aspirar.

Nuestras abuelas, por ejemplo, y sin conocer a las feministas europeas, lucharon contra las formas de dominación que les tocó vivir antes de la colonización de 1492. Nuestras abuelas no eran ningunas segundonas de sus hombres, ellas tenían mando político, militar y logístico en las luchas, como, nuestra abuela Bartolina Sisa, en las sublevaciones indígenas de 1871.

\section{¿ES LA MODERNIDAD OCCIDENTAL UN PARADIGMA REAL?}

Europa nos habla de modernidad y entre las cosas "modernas" a las cuales se refiere están los derechos de las mujeres. Creo que es 
necesario nos expliquen su modernidad y los derechos de las mujeres. Consideramos que Europa se pone una máscara de actualidad, entendiéndose como actualidad lo mejor que se produjo en la historia. Se pone esa máscara de modernidad, a través de ignorar el mundo que habitaba los territorios de Abya Yala, ignorando el cómo, al explotar estos nuestros territorios - trabajo y recursos naturales - luego toda esta riqueza fueron aprovechadas en usufructo de Europa, y gracias a ello la acumulación originaria del capital y el capitalismo fueron posibles. A propósito, evocando a Ignacio Sotelo, que dice:

Lo "moderno" evoca "ruptura" con el pasado. Como ha escrito Ignacio Sotelo, "implica la conciencia de una ruptura en la continuidad histórica: lo que fue ya no es; se viven tiempos nuevos". La modernidad es la ruptura con el mundo feudal. Su constitución, empero, no fue un hecho instantáneo, sino un largo proceso histórico en que los diferentes aspectos y dimensiones de la civilización occidental (eurocéntrica) y siguiendo cada uno sus propios ritmos y vicisitudes se fueron liberando poco a poco de sus ropajes feudales. En el plano de las ideas, por ejemplo, la modernización sólo puede considerarse constituida tras el acoplamiento de los movimientos del Renacimiento y de la Ilustración (Luís Garcia MARTIN, 2011, p. 266-267).

Europa también se ha encargado de significar y apropiarse de la palabra modernidad, como un supuesto, estadio mejor de toda la humanidad, considerando el tiempo como si fuera una línea donde Europa deja a todos los pueblos atrás, y se asumen como vanguardia "adelantada". Pero queremos cuestionar este supuesto estadio superior euroocidental, no es cierto, pues en el Kollasuyo - que era la región de lo que hoy es Bolivia - en 1492, cuando se dá este hecho colonizador, por ejemplo; la distribución administrativa respondía al poder del Inca pero también había autonomía en los territorios dominados y gobernados por autoridades locales, donde se tenía un control del hambre y la salud pública, por otro lado las mujeres, nuestras abuelas, participaban de la vida política y de las campañas militares autónomamente, cosa que en Europa esta participación no existía. Las mujeres todas en el Kollasuyu poseían tierra, medio tupu al nacer, hasta que se morían. Si damos un vistazo a Europa, las mujeres no tenian tierra y no participaban ni en 
la política ni en lo militar autónomamente. ¿Entonces quien estaba a la vanguardia, en cuanto a derechos en 1492?

El Feminismo euro occidental ha aportado en las luchas de las mujeres de Europa, eso no le vamos a negar, pero es bastante racista cuando se trata de mirar las luchas de las mujeres de otros continentes como el nuestro. Su eurocentrismo les lleva a imaginar que nos civilizaran, que solo ellas poseen los conceptos, que nos interpretaran. Que desde esos centros de poder e imaginarios, dictarán políticas para las mujeres a nivel mundial - bueno gracias al colonialismo y al imperialismo neoliberal - y eso es lo que hacen. Pero eso no quiere decir que, en verdad, nos interpretan, tampoco quiere decir que sus conceptos agotan el significado, de lo que las indígenas de Abya Yala somos.

"En su prólogo a la obra de Mohammed Abed Al-Yabri, Crítica de la razón árabe, Pedro Martínez Montávez afirma 'No hay un espacio en el que no se dé, individual y colectivamente, una actividad pensadora. Creer y mantener lo contrario es, sencillamente, una modalidad de racismo"' (Celia AMORÓS, 2004, p. 68).

El feminismo occidental como significado no nos comprende a las mujeres de nuestros territorios de Abya Yala - por si acaso tampoco pedimos que nos comprenda - y no nos comprenderá, en el sentido de abarcar y comprender, nuestra existencia. Presuponen que lo que ellas quieren, nosotras queremos o peor; que debemos querer. Hay coordinaciones que podemos hacer y sus demandas son parcialmente aceptables, para nosotras mujeres del sur, pues podemos estar de acuerdo con ellas, por ejemplo; en la lucha contra la violencia de los hombres hacia las mujeres, pero no vamos a estar de acuerdo con otras como la inclusión de las mujeres, en el sistema patriarcal, me explico. Por ejemplo; hay que luchar contra la violencia doméstica, pero también hay que luchar contra la violencia estructural que beneficia a las mujeres de los países occidentales, pues con nuestros trabajo y riquezas naturales se edifica el bienestar del norte rico. Es más, las mujeres de la clase media y de la burguesía, autonombradas feministas, viven privilegios a costa de nuestro trabajo, como mujeres en el sur y del trabajo de hombres de nuestros pueblos también. Las mujeres feminista y no feministas en Europa se incluyen en el Bienestar de sus Estados y ni una palabra con 
la expulsión de migrantes que buscan sobrevivir, o con los bombardeo de la OTAN a los pueblos árabes.

Hoy, en el contexto Latinoamericano, estas feministas con privilegios forman parte de la derechización de la sociedad. Un conjunto de feminismo burgués neoliberal, liberal y colonialista, son detractoras de procesos populares y socavadoras de gobiernos populares, con la excusa de que los proletarios, socialistas e indianistas son machistas. Claro que son machistas, como lo son los empresarios que son machistas de corbata, que explotan a las mujeres trabajadoras, les pagan menos y no pagan el trabajo doméstico, a costa de eso acumulan mas y más riqueza. Sin contar con el uso de prostitución, ni la trata y trafico de mujeres empobrecidas, el uso de mujeres en préstamos usureros de la banca y en el narco tráfico.

\section{LLAMARNOS FEMINISTAS ES TAMBIÉN UNA ESTRATEGIA SEMÁNTICA}

¿Por qué en medio de estas críticas que hacemos al feminismo, nosotras nos llamamos FeminismoComunitario?

Decíamos que, para bien o para mal, feminismo y feministas tiene un significado en el mundo, al cual le llamamos un campo semántico plantado, claro plantado hegemónicamente por la invasión desde Europa, pero es importante, a esta altura de nuestras luchas, posicionarse en el mundo y tomar decisiones ante este hecho. Por supuesto que nosotras nos hemos cuestionado, si era mejor elegir otro nombre para nuestras luchas y así no hacer el juego al eurocentrismo ciertamente, pudo ser una estrategia, ¡Claro que sí!. Pero elegimos otra.

Intentamos primero inventar otro nombre, para posicionarlo a lado del feminismo, y para ello fue necesario investigar, si antes otras mujeres tuvieron estos mismos cuestionamientos. Descubrimos que las feministas chicanas, allá por los años 70-80, se hicieron las mismas preguntas que nosotras y decidieron llamarse mujeristas, pero en la actualidad este nombre pocas personas lo conocen, tampoco se conoce esas luchas o se conoce muy poco. No queremos ser una anécdota, en este momento de la historia necesitamos posicionar otra manera de ver el mundo, porque el planeta tierra y la vida corren peligros muy 
grandes. Es tiempo propicio para cuestionar todas las hegemonías y todos los poderes conocidos.

Otro hecho que nos hizo reflexionar, sobre si llamarnos feministas o no, es aquel que se refiere a la relación entre dos conceptos, Cosmovisión indígena y Filosofía. Cuando hablamos con nuestros hermanos indígenas, ellos dicen que la cosmovisión indígena es mucho más que la filosofía occidental. Puede ser, sin embargo, eso ¿quién lo conoce y reconoce como un hecho? Nadie, la filosofía sigue siendo la que manda. Nosotras vemos que no es cuestión de voluntarismo, esto del manejo de las relaciones semánticas. Entendemos por relaciones semánticas a las que tienen que ver con los aspectos jerárquicos del significado, el sentido o interpretación de signos, ya sean lingüísticos, como símbolos, palabras, expresiones o representaciones formalizadas. No es cuestión de voluntarismo, hay una colonización del lenguaje, es un hecho. Entonces no es cuestión de cerrar los ojos y decir que el colonialismo no nos va a "comer". Nos fagocitan desde las academias.

Por mucho esfuerzo y buena voluntad que le pongamos, por ejemplo, a las cosmovisiones indígenas, y con ese nombre, desde las hegemonías del pensamiento, las interpretaciones van a privilegiar siempre al significado eurocéntrico. Las relaciones son más bien al revés de lo que suponen y dicen nuestros hermanos indianistas, respecto de filosofía. Las cosas de indios son cosmovisión y el pensamiento "culto", es europeo, por tanto; es filosofía,

Consecuencia de esa aseveración es que los indios no son filósofos o peor, los indios no pueden ser filósofos, solo alcanzan a tener y hacer cosmovisiones. Lo mismo acontece con el arte, que es una actividad que se desarrolla bajo los parámetros de la cultura occidental, en vez nuestros trabajos estéticos, que se desarrollan bajo nuestras sensibilidades estéticas, son llamados artesanías.

Ese es el mundo en el que vivimos, nosotras, después de reflexionar, decidimos entrar en su cancha y pelearles espacios semánticos, llamándonos feministas también, y así de igual a igual cuestionar el significado euro céntrico de este término, generando un espacio para todas las mujeres del mundo, para reconocer nuestros cuerpos y nuestras luchas. Por supuesto que también para nuestras luchas en Bolivia y nuestro proceso político de cambio. 
Nosotras, feministas comunitarias, elegimos la estrategia de pelear el significado colonizador y pelear las atribuciones conceptuales, del concepto Feminismo y Feminista, desde ahí debatir de que feminismo estamos hablando o sea que, significado, le estamos dando al feminismo.

Nosotras, al nombrarnos FeministasComunitarias, no imitamos a Europa y EEUU, desafiamos la soberbia, convocamos al dialogo y en su propio campo semántico les disputamos el contenido. Celia Amorós, feminista española, dice:

En efecto imitar el espíritu crítico de la potencia colonizadora que-teóricamente, al menos - exporta e induce ilustración no haría sino demostrar por parte de la potencia receptora que carece de su propio espíritu crítico, ya que - acríticamente se viene a imitarlo (Celia AMORÓs, 2004 p. 69).

Nosotras del FeminismoComunitario no imitamos, ni solo criticamos, convocamos y disputamos el campo semántico, al feminismo occidental, y creamos conceptos que aportan ha nuestros pueblos, porque entendemos que una propuesta, un pensamiento, sino está vivo, sino responde y propone, ante los desafíos del mundo de hoy, que es enfrentar un sistema desde distintos lugares y al mismo tiempo, pues será absorbido por el propio sistema y morirán sus esperanzas.

Llamarnos feministas es dejar la puerta abierta, a la posibilidad de construir un movimiento mundial de mujeres para construir la Comunidad de comunidades. Es el reconocimiento de la posibilidad, de coordinación de las luchas, unas con otras, más bien partir de construir con todas las mujeres, sin jerarquías ni privilegios.

\section{QUE NOS VAN A ENSEÑAR QUE NO SEPAMOS}

Las políticas públicas de los gobiernos, los programas de las ONGs, los discursos de las iglesias, son miradas colonizadoras, tienen una visión de nosotras, las mujeres indígenas, como si fuéramos las eternas ignorantes, las tontitas, las incivilizadas, o - en el "mejor" de los casos - somos las ingenuas e inocentes. No es desde la soberbia que decimos y aseveramos esto, aunque suene muy duro, nuestras abuelas nos enseñaron el respeto, pero también la indignación cuando ya llegan 
a colmos. Este es el caso de cómo andan las relaciones entre mujeres, especialmente en el espacio del feminismo euro centrado, que continua e insisten en posicionarse como paradigma y dirección política de todas las luchas de las mujeres en el mundo. Son las autodenominadas políticamente correctas, el resto tendríamos miles de defectos y pretenden interpretarnos desde sus categorías moralistas y reduccionistas. Si, probablemente tenemos muchos defectos, la pregunta es: ¿Y quién no?

Ya el feminismo comunitario redefinió el feminismo como espacio de encuentro y rendición de cuentas entre mujeres, sin embargo el feminismo eurocentrado en todas sus versiones - incluidas anarquistas y autónomas - que se esfuerzan por expulsar de la discusión a quien no esté eurocentrada. Pretextos para ello no les faltan, desde decir que no hay nada nuevo bajo el sol, pues el pensamiento eurocentrado ya pensó en lo que nuestros cuerpos son y sienten. Pasando por legitimar plagios, hasta servirse de calumnias y difamaciones, contra el FeminismoComunitario. Es claro que afectamos sus intereses en lo más profundo, pero no deja de indignarnos escuchar las catalogaciones y opiniones sobre nosotras, FeministasComunitarias. Existe la convicción de que las mujeres indígenas no nos hubiéramos dado cuenta, o no pudiéramos por nosotras mismas darnos cuenta de nuestras situaciones de vida y que fueran las ONGs de la equidad de género o los feminismos euroccidentales, los que van liberarnos, las que van a decirnos que somos personas.

Hay condiciones precisamente históricas para que nosotras como indígenas estemos como estamos. Las Feministas comunitarias explicamos este hecho a través de lo que llamamos el Entronque Patriarcal, (Julieta PAREDES, 2010) entronque de dos patriarcados el colonial y el patriarcado indígena, que a partir de 1492 se entroncan, realizando una serie de pactos entre hombres, relaciones que configuran la actual situación de las mujeres indígenas, de mayor opresión y discriminación. Pero a la vez reivindicamos nuestras propuestas, que hoy es uno de los logros que tiene, el FeminismoComunitario, es la propuesta de despatriarcalizacion, como la palabra y la propuesta de mundo de las mujeres desde las luchas ancestrales (Julieta PAREDES, 2016).

Ciertamente hay información que por ahora no sabemos ni conocemos, pero cuando estamos hablando de la conciencia de nuestra digni- 
dad, del auto reconocimiento, que somos gente jaq'e -como decimos en aymara - ¡Claro que sabemos quiénes somos y lo que queremos! Nadie nos va a enseñar lo que ya sabemos, desde nuestros cuerpos.

Claro que sabemos que nos duele que nos golpeen, que nos humillen y maten, nuestros propios hermanos y compañeros, pero como resolvemos eso no lo van a decidir desde afuera de nosotras, desde una neocolonizacion o colonización feminista de nuestros cuerpos, el destino de nuestros cuerpos lo vamos a decidir nosotras en nuestras organizaciones y comunidades. Luchando al mismo tiempo contra las injusticias que vivimos; los hombres, mujeres, personas intersexuales y la naturaleza por parte de un sistema de dominio. $\mathrm{Y}$ al mismo tiempo peleando contra el machismo y la violencia en nuestras comunidades y aldeas.

No esperamos que entiendan esto las ONGs, los colectivos de feministas eurocentradas, las académicas e intelectuales feministas y la propia izquierda, pero podrían hacer el esfuerzo, mínimamente reflexionar que las mujeres indígenas, somos mujeres y sabemos que lo somos. Sabemos lo que queremos y no ponerse de jueces de lo que no conocen, pues desconocen nuestras estrategias. ¡Hay mucha soberbia! Creemos que debe plantearse el respeto como base de la relación, sin embargo, esto no se da, con el pretexto de que nuestros hermanos indígenas serían unos salvajes y nosotras cómplices o igualmente salvajes que ellos. Nos damos cuenta como está considerada a menos nuestra voz, para convertirnos en víctimas tuteladas, y hay algunas indígenas que les hacen el juego a estos conceptos y son las reconocidas, pues cumplen el papel que se espera de nosotras las indígenas, que seamos las que nos quejamos, las víctimas y no las que decidimos, no las que nos defendemos y luchamos por nuestro territorio cuerpo y nuestra tierra y territorio.

Me llaman la atención las muchas, investigaciones que se hacen sobre nosotras las mujeres indígenas y de nuestros pueblos originarios, pero ciertamente no conozco ninguna investigación sobre mujeres blancas, o sobre mujeres académicas o mujeres feministas de los partidos burgueses o no, sobre, por ejemplo, la violencia que los hombres blancos y burgueses ejercen sobre ellas y lo que ellas hacen al respecto. Podrían ser buenos objetos de investigación, sugerimos, el fascismo y 
derechización de las mujeres, o el racismo entre mujeres y el racismo de mujeres y hombres de las clases medias. Puedo "pecar" de ignorante, pero que yo sepa las luchas y las denuncias de violencia, las damos las mujeres de a pie, las indígenas, las negras, las empobrecidas. Las mujeres intelectuales, de las ONGs y mujeres políticas de los partidos, hablan a nombre de las pobres mujeres que sufren violencia y no hablan de ellas pues, hasta el momento, ellas no se reconocen "víctimas" pero son presurosas y están a la caza de mujeres indígenas o empobrecidas que se declaran víctimas.

Esta es una nueva relación muy perversa expresada hoy por ejemplo en el boom mediático y financiable de "Ni Una Menos" que está en todos los medios de comunicación internacionales, pero no están ocupando ese mismo espacio, la lucha de las mujeres indígenas contra el extractivismo y la depredación de nuestros territorios o las muertes de miles de mujeres y hombres, niñas y niños indígenas, en la lucha por la recuperación de tierra y territorio, en la "Demarcación Ya" en Brasil. En contraposición a las mujeres feministas políticamente correctas, estamos las mujeres en las luchas de nuestros pueblos que no tienen la cobertura mediática. Porque - lo digo irónicamente - somos un puñado de "salvajes", que luchamos al mismo tiempo contra la violencia de quienes envenenas la madre naturaleza y contra la violencia sobre nuestros cuerpos.

\section{LA ACADEMIA HOY}

Creo necesario señalar que el interés por nuestros pueblos originarios, en lo que se refiere investigaciones académicas, debería ser un espacio para la discusión, la reflexión y el cuestionamiento. Y con el riesgo de las susceptibilidades que suelen bloquear los espacios de reflexión, queremos manifestar que en general y en particular, hasta el momento, no conocen o no consideran importante, por ejemplo, el aporte histórico que hace el FeminismoComunitario, pocos son los espacios, como esta revista Mandrágora, abierta a la comprensión del lugar de las mujeres indígenas, en las historias del mundo.

El feminismo comunitario ya planteó un camino de comprensión de nuestras historias de luchas en el libro "Hilando Fino" el año 2009, que inicia el aporte escrito en libros, del FeminismoComunitario, soporte de 
documento, que va a cuestionar no sólo las hegemonías, sino las luchas y el proyecto de mundo que hoy se debate.

\section{LA MATERNIDAD}

La maternidad, para el FeminismoComunitario, no deja de ser el intento de entender el camino que hacemos las mujeres indígenas de descolonización de nuestros cuerpos y las prácticas y pensamientos que de estos nuestros cuerpos emanan. Por eso es valioso ser mujer y ser mujer que lucha en el ámbito de que lo personal es político.

Sin duda que la maternidad y el cuidado de la vida de las niñas y niños en comunidad es cuestionar lo parámetros individualistas de una sociedad, de la propiedad privada de los cuerpos, de las wawitas (niñas y niños) como decimos en aymara. Este hecho de partida significa cuestionar la violencia normadora de los cuerpecitos, que también en nuestras comunidades se dan, con las exigencias de parámetros de masculinidad y feminidad, que también son impuestos a niños y niñas, eso es lo que nosotras hemos llamado la relación de genero indígena y por eso mismo opresora en construcción, de las relaciones entre hombres y mujeres indígenas. Cierto que las comunidades indígenas traen valores y es importante potenciarlos, pero también es imprescindible asumir una postura crítica al respecto de prácticas que reproducen relaciones jerárquicas y violentas.

Son responsabilidades de la comunidad garantizar la vida que crece, pero eso no significa que en última instancia sean los padres y madres, que reclamen el cuerpo del hijo o hija, para beneficio económico de la familia, son los cruces presentes en nuestras prácticas sociales, donde se mezclan las ideologías, pensamientos y decisiones que tomamos mujeres y hombres indígenas sobre como criar a nuestras hijas e hijos.

Eso es lo que debemos cuestionarnos en cuanto se refiere a las propuestas que tenemos como indígenas; es cómodo reclamar el cuerpo de las wawas para el trabajo de la familia, pero cuan solas y solos se sienten las madres y los padres de los 43 de Ayotzinapa, o las madres de desaparecidas y desaparecidos, al momento de reclamar los cuerpos de sus wawitas luchadoras. O cuan solas se sienten las mamás de las hijas que fueron asesinadas por los hombres machistas y femicidas. Las niñas y niños son de las comunidades también. 


\section{LAS ESPIRITUALIDADES}

La colonización nos trajo un Dios hombre, extraño, que hacia un radical contraste con la diversidad de energías espirituales que circulaban en nuestros territorios, energías que eran y son todavía motivo de los ritos que nos conectan, con una trascendencia de la vida de nuestra Madre Naturaleza.

Recogemos todos los días, en algunas comunidades especialmente de la amazonia, una espiritualidad para la vida, que se expresa en el agradecer a la Madre Agua, la Madre tierra, la Madre Iluvia, nuestra existencia. La colonización de lo sagrado nos llevó por el reduccionismo del monoteísmo patriarcal. Que contribuye a la jerarquía machista de la vivencia de lo sagrado, así dejamos de lado lo sagrado, de las múltiples relaciones entre la humanidad, y lo sagrado en las relaciones de la humanidad con la naturaleza

En la colonización de nuestros cuerpos y territorios, los defensores de indio como Fray Bartolomé de las Casas, miro a los indios principalmente, no a las indias. Hecho que se vuelve a repetir en la llamada teología de la liberación, que miran en su "Opción por los pobres" a los pobres y no a las pobres. Para el pensamiento religioso, las mujeres fueron objeto de su labor evangelizadora, pero siempre como segundonas después de los hombres, lo hombres indígenas, por ejemplo, fueron reconocidos como ayudantes de curas y pastores, las mujeres no.

Que diferente era la vida de nuestras abuelas antes de la colonización, donde ellas eran las responsables de los ritos de Paxsi mama, o Madre Luna y los abuelos del Tata Inti o Padre sol. Diferente la vida de las mujeres, con respecto a lo sagrado, ritual y espiritual, cuando el reconocimiento social, se expresaba a través de un lugar igualitario, para las mujeres, en los territorios del Kollasuyo. No vamos a decir que la vida de nuestras tatarabuelas era un paraíso, pero ciertamente estaban mucho mejor, que lo que estamos ahora como mujeres indígenas. ¿Qué fue lo que paso? Pues, aconteció, la colonización y el Entronque Patriarcal.

\section{CONCLUSIONES}

El presente escrito es una pincelada introductoria de lo que es el pensamiento, que el movimiento y la organización social llamada: 
FeminismoComunitario de Abya Yala. tiene. El FeminismoComunitario inicia un camino de descolonización del feminismo, que originalmente se plasmó en el libro Hilando Fino, donde reconceptualizamos conceptos como feminismo, género y patriarcado, así como su utilidad para las luchas de nuestros pueblos. También nos parece útil dejar claras las diferencias, de nuestras raíces de pensamiento, conocimiento y práctica social, de lo que es Europa y los feminismos europeos, para poder así hablar de la creación de un pensamiento propio.

\section{REFERENCIAS}

AMORÓS, Celia. Por una ilustración multicultural. Quaderns de filosofía i ciencia, n. 43, 2004, p. 67-79. Disponible en: https://www.uv.es/sfpv/quadern_textos/v34p67-79.pdf>.

MARTIN, Luís Garcia. La polémica en torno a la legitimidad del derecho penal moderno. Revista Jurídica. Universidad Católica de Santiago de Guayaquil, 2011, p. 265-335. Disponible en: https://www.revistajuridicaonline.com/wp-content/uploads/2011/04/29_265a336_lapolemica.pdf>.

PAREDES, Julieta. Hilando fino, desde el feminismo comunitario. La Paz: Mujeres Creando Comunidad, 2010.

PAREDES, Julieta. El desafío de la Despatriarcalizacion. La Paz: FeminismoComunitario de Abya Yala, 2016.

Submetido em: 2-12-2018

Aceito em: 13-12-2018 